\title{
ERRATUM
}

\section{Erratum to: Long-term and short-term predictors of worries about getting Alzheimer's disease}

\author{
Stephen J. Cutler ${ }^{1,2} \cdot$ Corina Brâgaru ${ }^{2}$
}

Published online: 15 September 2015

(c) Springer-Verlag Berlin Heidelberg 2015

\section{Erratum to: Eur J Ageing \\ DOI 10.1007/s10433-015-0350-3}

Unfortunately, in Table 4 the value of worry for the variable 'Interaction between personal familiarity and beliefs' was mentioned incorrectly.

The correct value is updated in Table 4.
Table 4 Standardized coefficients (standard errors in parentheses) in the prediction of worry about getting Alzheimer's disease

\begin{tabular}{|c|c|}
\hline Variable & Worry \\
\hline Self-rated memory intercept factor & $\begin{array}{l}-\mathbf{0 . 2 6 4} * \\
(0.107)\end{array}$ \\
\hline Self-rated memory slope factor & $\begin{array}{l}-\mathbf{0 . 2 0 8} * * * \\
(1.787)\end{array}$ \\
\hline Self-rated memory quadratic factor & $\begin{array}{l}-\mathbf{0 . 2 3 4} * \\
(32.817)\end{array}$ \\
\hline Personal familiarity & $\begin{array}{l}0.051 \\
(0.180)\end{array}$ \\
\hline Beliefs & $\begin{array}{l}\mathbf{0 . 0 7 3} * * \\
(0.081)\end{array}$ \\
\hline $\begin{array}{l}\text { Interaction between personal } \\
\text { familiarity and beliefs }\end{array}$ & $\begin{array}{l}\mathbf{0 . 0 8 3}^{+} \\
(0.220)\end{array}$ \\
\hline Age & $\begin{array}{l}-\mathbf{0 . 1 3 6} * * * \\
(0.003)\end{array}$ \\
\hline Marital status & $\begin{array}{l}0.021 \\
(0.075)\end{array}$ \\
\hline Gender & $\begin{array}{l}0.005 \\
(0.092)\end{array}$ \\
\hline Education & $\begin{array}{l}0.035 \\
(0.027)\end{array}$ \\
\hline Depression & $\begin{array}{l}\mathbf{0 . 0 9 2} * * \\
(0.021)\end{array}$ \\
\hline Health & $\begin{array}{l}0.001 \\
(0.038)\end{array}$ \\
\hline$R^{2}$ & 0.105 \\
\hline
\end{tabular}

The online version of the original article can be found under doi: 10.1007/s10433-015-0350-3.

\section{Stephen J. Cutler}

scutler@uvm.edu

1 Department of Sociology, University of Vermont, Burlington, VT 05405, USA

2 Faculty of Sociology and Social Work, University of Bucharest, 9 Schitu Măgureanu Street, Bucharest, Romania 